\title{
A Hybrid Spectral/Finite-Volume Algorithm for Large-Eddy Simulation of Scalars in the Atmospheric Boundary Layer
}

\author{
Marcelo Chamecki • Charles Meneveau • \\ Marc B. Parlange
}

Received: 25 February 2008 / Accepted: 21 July 2008 / Published online: 5 August 2008

(C) Springer Science+Business Media B.V. 2008

\begin{abstract}
Pseudospectral methods are frequently used in the horizontal directions in large-eddy simulation of atmospheric flows. However, the same approach often creates unphysical oscillations for scalar fields if there are horizontal heterogeneities in the sources and/or sinks, as is usual in air pollution problems. A hybrid approach is developed to combine the use of pseudospectral representation of the velocity field and bounded finite-volumes for the scalar concentration. An interpolation scheme that yields a divergence-free interpolated velocity field is derived and implemented, and its importance is illustrated by two sample applications.
\end{abstract}

Keywords Conservative velocity interpolation - Divergence free · Finite volumes · Large-eddy simulation · Passive scalars

\section{Introduction}

Spectral methods have been extensively used in large-eddy simulation (LES) and direct numerical simulation (DNS) of turbulent homogeneous flows. The high accuracy coupled with variance conserving properties of spectral methods are major advantages over other discretization methods (Canuto et al. 1988). A common approach for LES of the atmospheric

\footnotetext{
M. Chamecki

Department of Geography and Environmental Engineering and Center for Environmental and Applied Fluid Mechanics, Johns Hopkins University, Baltimore, MD, USA

e-mail: chamecki@jhu.edu

C. Meneveau $(\varangle)$

Department of Mechanical Engineering and Center for Environmental and Applied Fluid Mechanics, Johns Hopkins University, Baltimore, MD, USA

e-mail: meneveau@jhu.edu

\section{B. Parlange}

School of Architecture, Civil, and Environmental Engineering, Ecole Polytechnique Fédérale de Lausanne, Lausanne, Switzerland e-mail: marc.parlange@jhu.edu
} 
boundary layer (ABL) introduced by Moeng (1984) is to combine pseudospectral representation in the horizontal directions and second-order centred finite-differences in the vertical non-homogeneous direction. This combination takes advantage of spectral accuracy in the homogeneous directions and allows flexibility for boundary conditions in the vertical direction and is widely used in a variety of ABL applications (e.g. Khanna and Brasseur 1997; Albertson and Parlange 1999; Kosovic and Curry 2000; Kumar et al. 2006; Basu et al. 2007; Mao et al. 2007; Yue et al. 2007).

In many cases, such as air pollution, one is also interested in the turbulent transport of scalar quantities. It is often the case that, although the flow is horizontally homogeneous, the scalar sources are not. If spectral representation is also applied to the scalar conservation equation, unphysical solutions such as negative concentrations often develop. To avoid these problems, the usual approach is to use bounded schemes based on finite-difference or finitevolume discretizations for the velocity and scalar equations (e.g. Nieuwstadt and de Valk 1987; Xie et al. 2004). Another option, to be developed here, is a hybrid approach where the momentum equations are discretized using the spectral method and the scalar conservation equation is solved on a finite volume grid. The idea is to combine the high accuracy and kinetic energy conservation properties of spectral representation for the velocity field with the physically motivated bounded properties for the scalar field (preserving high accuracy for the velocity field is important given the large impact of advection on the evolution of the scalar field).

A critical component of coupling the two different discretization methods is the interpolation of the divergence-free velocity field to the finite-volume surfaces. It is essential that the interpolated velocity field preserves the divergence-free character locally on the new grid to high precision (Carey et al. 2001).

There are several methods documented in the literature regarding interpolation of divergence-free vector fields. In Carey et al. (2001), a finite-element solution of the velocity field is coupled with a coarser finite-volume water quality model. They proposed a solution based on Lagrangian multipliers to enforce the local divergence-free constraint on the finitevolume grid. Their approach is formulated as a global saddle-point optimization for the interpolated field. Similar problems are also encountered in adaptive mesh refinement schemes for magnetohydrodynamics where the problem is usually solved by constructing divergencefree interpolation formulae (Balsara 2001; Li and Li 2004). Another approach proposed by Bochev and Shashnov (2005) for deforming (moving) finite-element grids is based on reconstructing a vector potential associated with the velocity field and then interpolation of this potential.

In this paper we propose a new interpolation scheme to couple a spectral representation of the velocity field with a finite-volume solution to the advection-diffusion equation. Unique to this problem is the fact that on the original grid the divergence-free condition is enforced based on the nonlocal spectral derivatives while on the new target grid the interpolated velocity is required to satisfy the same constraint in a local discretization. The method takes advantage of specific characteristics of both grids, yielding a simple and efficient algorithm without the requirement of Lagrangian multipliers or vector potential reconstruction. Although the method is specific to this original/target grid choice, this is a combination that may have wide applications in fluid dynamics.

As an example, the new method is applied to a LES of the neutrally buoyant atmospheric boundary layer. Simulations are carried out using the proposed conservative interpolation scheme, and results are compared with trigonometric interpolation. A brief description of the numerical code that motivates the present development is provided in Sect. 2 and the new interpolation scheme is presented in Sect. 3. Results and conclusions are presented in Sects. 4 and 5. 


\section{LES Code Description}

The numerical discretization used herein follows the approach used by Moeng (1984) and combines a pseudospectral representation in the horizontal directions and second-order centred finite differences in the vertical. The vertical component of the velocity field $w$ is placed on a staggered grid as shown in Fig. 1a. The second-order Adams-Bashforth scheme is used for time advancement, and the filtered momentum equations are solved in a rotational form with a constant mean streamwise pressure gradient forcing. The flow is assumed to be incompressible and molecular viscosity is neglected due to the high Reynolds number. The subgrid-scale (SGS) stress tensor is modelled using a Smagorinsky-type model (Smagorinsky 1963), where the coefficient is determined using the Lagrangian-averaged scale-dependent dynamic approach of Bou-Zeid et al. (2005). Stresses are imposed at the bottom boundary based on a local application of the logarithmic law as described in Bou-Zeid et al. (2005). More details on the solution of the velocity field can be found in Albertson and Parlange (1999).

The same numerical model used here has been used by Tseng et al. (2006) to model pollutant dispersal from a point source in Baltimore city, U.S.A. However, Tseng et al. (2006) also used a pseudospectral representation for the passive scalar field and, in order to avoid negative concentrations due to the Gibbs phenomenon, the turbulent diffusivity had to be increased by a factor of 20 . To further illustrate the problem, we use a pseudospectral representation to simulate the dispersal of a passive scalar from a point source in the neutral ABL. The approach is similar to the one used by Tseng et al. (2006), except that the standard value for the Schmidt number $(S c=0.4)$ is adopted here. An instantaneous $x z$-cut of the concentration field and its time-averaged counterpart are shown in Fig. 2. Clear spots of negative concentration can be observed in both figures. Note that the Gibbs phenomenon also induces large unphysical concentrations upwind from the source location.

In order to avoid problems such as those illustrated in Fig. 2, we couple the pseudospectral solution for the velocity field with a finite-volume discretization for the advection-diffusion equation. The finite-volume grid is placed in such a way that the centre of the volume where the concentration field $C_{i, j, k}$ is stored is located at the velocity nodes. The arrangement, as well as the location where each velocity component is required for the evolution of the scalar field, is shown in Fig. 1b. For clarity, velocity components on the spectral grid
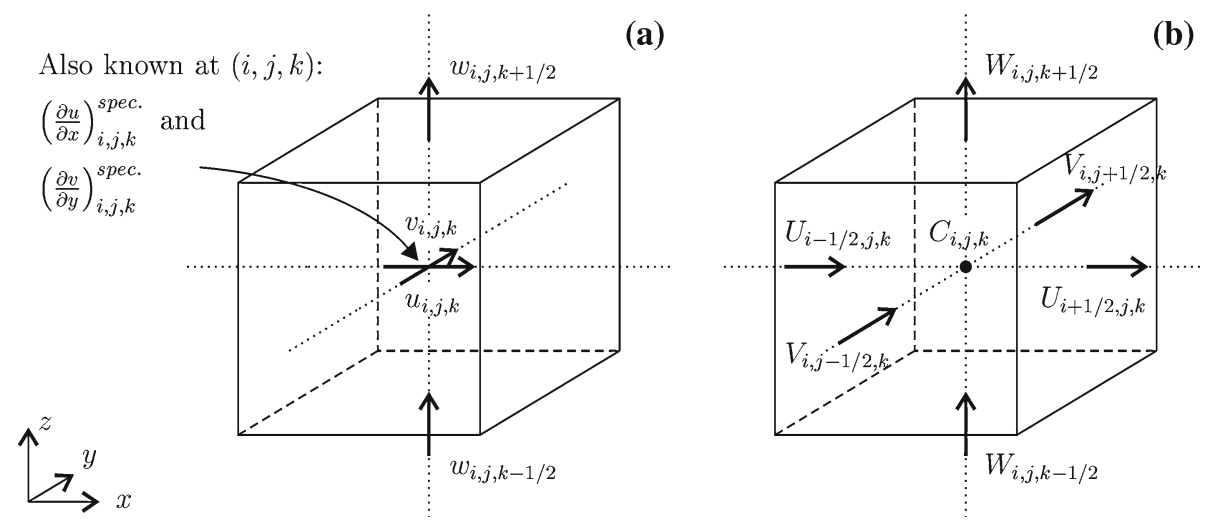

Fig. 1 Discrete velocity locations (a) in the pseudospectral representation and (b) needed for the finite-volume discretization 


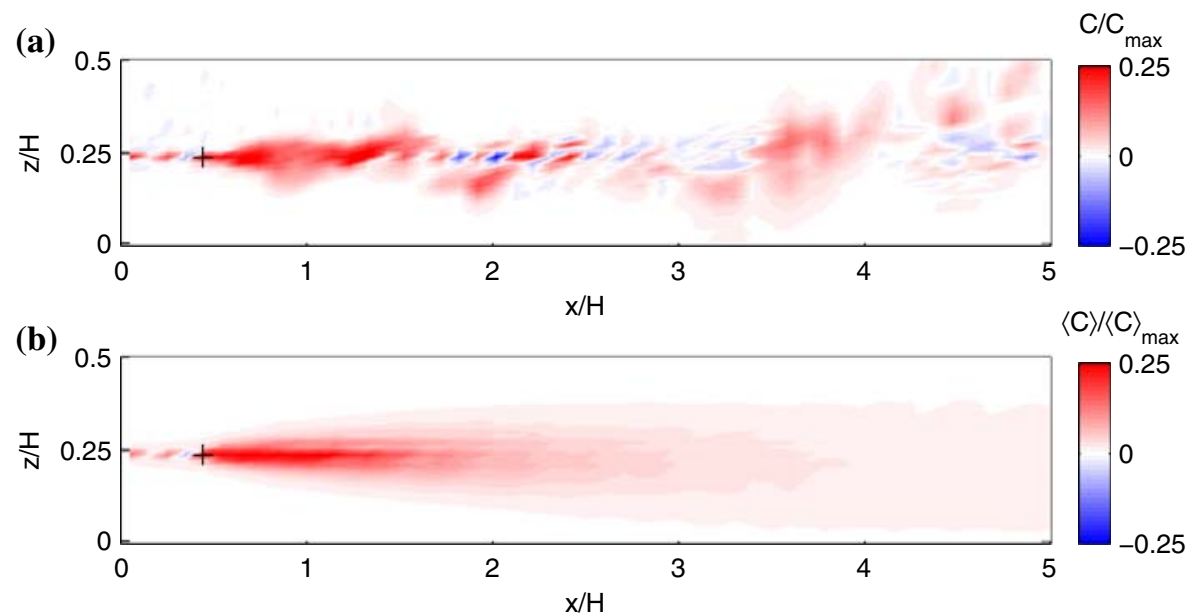

Fig. $2 x z$-cut of simulation of a point-source emission using spectral representation: (a) instantaneous concentration and (b) time-averaged concentration over $2.7 \times 10^{4}$ steps $(3 \mathrm{~h})$. The cross indicates the location of the source

will be represented by lower case $(u, v, w)$ and their interpolated counterpart on the finitevolume faces will be represented by upper case $(U, V, W)$. As can be seen in the figure, due to the vertical staggered grid, no interpolation for the vertical component is required. For the horizontal directions trigonometric (exact spectral) interpolation and the conservative interpolation proposed in the next section are implemented. The SGS term in the filtered advection-diffusion equation is modelled using a flux-gradient relation where the eddy diffusivity is obtained by dividing the Lagrangian-averaged scale-dependent eddy viscosity $\left(v_{s g s}\right)$ by a constant Schmidt number, as is usual practice in simulations of the atmospheric boundary layer (e.g. Andren et al. 1994). The advection term is computed using the bounded third-order upwind interpolation scheme SMART (Gaskell and Lau 1988). The implementation follows the method suggested by Waterson and Deconinck (2007).

\section{Conservative Interpolation}

The main motivation for the proposed interpolation scheme is the fact that in a spectral representation conservation of mass is enforced using the spectral (nonlocal) derivatives. In general, the velocity field interpolated to the surfaces of the finite volumes will not satisfy the continuity equation. In other words, if the velocities $(U, V, W)$ are obtained by linear or trigonometric interpolation of $(u, v, w)$, the conservation of mass on the finite-volume grid

$$
\begin{aligned}
& \left(U_{i+1 / 2, j, k}-U_{i-1 / 2, j, k}\right) \Delta y \Delta z \\
+ & \left(V_{i, j+1 / 2, k}-V_{i, j-1 / 2, k}\right) \Delta x \Delta z \\
+ & \left(W_{i, j, k+1 / 2}-W_{i, j, k-1 / 2}\right) \Delta x \Delta y=0
\end{aligned}
$$

will, in fact, not be satisfied. It is well known that this has important consequences for the evolution of the scalar equation, as will also be illustrated in the next section. For now, we propose an interpolation scheme where Eq. 1 is exactly satisfied. 
It is important to note that continuity is enforced on the spectral derivatives. The solution to the problem can be simply obtained if: (i) the spectral derivatives are used instead of the spectral velocity field $(u, v, w)$, and (ii) the derivatives are integrated using a scheme consistent with the finite-volume discretization. The procedure is illustrated for the code described in the previous section, in which only the horizontal components need to be interpolated. Generalization to fully three-dimensional spectral codes is trivial.

The solution proposed is to calculate $U$ and $V$ from the spectral derivatives $(\partial u / \partial x)^{\text {spec. }}$.

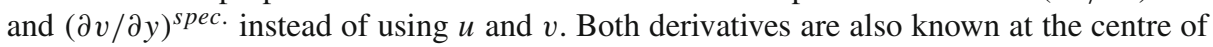
the finite volumes and they satisfy continuity exactly:

$$
\left(\frac{\partial u}{\partial x}\right)_{i, j, k}^{\text {spec. }}+\left(\frac{\partial v}{\partial y}\right)_{i, j, k}^{\text {spec. }}+\frac{w_{i, j, k+1 / 2}-w_{i, j, k-1 / 2}}{\Delta z}=0 .
$$

If we use a second-order centred discretization (which is consistent with the finite volume discretization), then the velocity field can be obtained from:

$$
\begin{aligned}
& \frac{U_{i+1 / 2, j, k}-U_{i-1 / 2, j, k}}{\Delta x}=\left(\frac{\partial u}{\partial x}\right)_{i, j, k}^{\text {spec. }} \\
& \frac{V_{i, j+1 / 2, k}-V_{i, j-1 / 2, k}}{\Delta y}=\left(\frac{\partial v}{\partial y}\right)_{i, j, k}^{s p e c .}
\end{aligned}
$$

It is obvious that if the velocity field $(U, V)$ is determined from the above equations, the finite-volume conservation of mass given by Eq. 1 is exactly satisfied.

However, Eq. 3 for each element $(i, j, k)$ has two unknowns and additional information is required to completely determine the solution. Since the face velocity obtained has to be the same for both elements sharing the common face, the solution for each pair of neighbouring elements is coupled. If Eq. 3 is written for all elements in the two-dimensional horizontal plane, the solution can be arranged in $N_{y}$ decoupled systems of $N_{x}$ equations for the $U$ elements, each system corresponding to one line along the $x$ direction (the last equation is obtained taking into account the periodicity of the velocity field). In the same way, one obtains $N_{x}$ systems with $N_{y}$ equations for the $V$ elements. However, all these systems are undetermined, since the equation from the periodicity is a linear combination of two other equations (obviously, one cannot fully determine the velocity field by imposing only the derivatives everywhere). One more equation has to be added. We choose to impose the average velocity along the line, by averaging the original field $(u, v)$ along the same line. As an example, the final system of equations for the $(j, k)$ line of $U$ is given by:

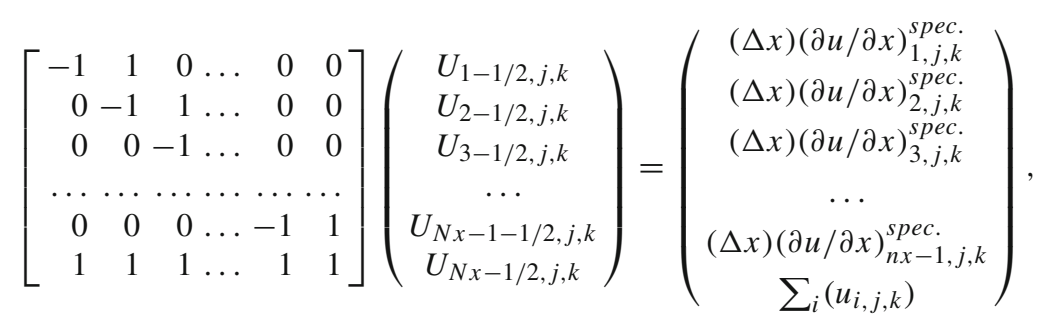

and similarly for $V_{i, j-1 / 2, k}$.

The solution for the system $A x=b$ above can be implemented in an efficient algorithm by noting that: (i) all the matrices for each velocity component are the same, and (ii) the structure of each matrix is very simple and its inverse can be easily determined. The inverse 
of the matrix $A$ depends only on $N$, and it was evaluated for several values of $N$. The results were observed to be of the form:

$$
A^{-1}=\frac{1}{N}\left[\begin{array}{rrrrr}
(1-N) & (2-N) & (3-N) & \ldots(N-1-N) & 1 \\
1 & (2-N) & (3-N) & \ldots(N-1-N) & 1 \\
1 & 2 & (3-N) & \ldots(N-1-N) & 1 \\
1 & 2 & 3 \ldots(N-1-N) & 1 \\
\ldots & \ldots & \ldots \ldots r & \ldots \\
1 & 2 & 3 \ldots(N-1-N) & 1 \\
1 & 2 & 3 \ldots & (N-1) & 1
\end{array}\right] .
$$

Multiplication by $A$ yields the identity matrix as can be seen by inspection. The proposed scheme is then reduced to simply calculating the spectral derivatives and assembling the vectors on the right-hand side of Eq. 5. The required velocity components are then obtained by multiplying the vectors by the matrix $A^{-1}$ given above. Note that each velocity component is determined independently from the others (i.e. in the interpolation algorithm the velocity components are decoupled). The final algorithm is fast and the number of operations required for a simulation with $N_{t o t}=N_{x} \times N_{y} \times N_{z}$ can be estimated as follows: for a given velocity component, say $U$, each line along that velocity component's direction (in this case $x$ ) will require one matrix-vector multiplication (order of $N_{x}^{2}$ operations). The interpolation of $U$ will require $N_{y} N_{z}$ matrix-vector multiplications and the total number of operations required is of order $N_{x}^{2} N_{y} N_{z}$. If $N_{x}=N_{y}=N_{z}=N$, the number of operations required for $N_{t o t}=N^{3}$ points is of order $N^{4}$ and the total number of operations required is of order $N_{\text {tot }}^{4 / 3}$ (the fastest method would be linear interpolation which, in this case, would require $N_{\text {tot }}$ operations).

\section{Sample Results}

Both trigonometric and conservative interpolations were implemented in the LES code described in Sect. 2. In both cases the velocity field is calculated using a pseudospectral representation and the passive scalar field is calculated on a finite-volume grid (the only difference being the interpolation scheme used to obtain the velocity field needed to evolve the advection-diffusion discretized equation). As noted by Yeung and Pope (1988), trigonometric interpolations can be considered "exact" on the truncated basis. The comparisons presented here are based on $64^{3}$ simulations with domain size $2 \pi H \times 2 \pi H \times H$ and imposed pressure gradient $(1 / \rho)|\nabla p|=u_{*}^{2} / H$, where $u_{*}$ is the friction velocity. The velocity field is evolved until statistical steady-state is reached $\left(9 \times 10^{4}\right.$ timesteps $)$ and then the scalar field is "turned-on" and integrated for another $2 \times 10^{5}$ timesteps. In Fig. 3 the two interpolations are compared for the instantaneous streamwise component of velocity, one at the first vertical level and the other halfway between the lower boundary and the centre of the domain $\left(N_{z} / 4\right)$. The two interpolation schemes yield similar results, the differences being larger closer to the wall, where the velocity field is less smooth.

Two sets of simulations are carried out with each interpolation scheme in order to demonstrate the importance of the conservative interpolation. The first set is carried with zero scalar fluxes at the top and bottom boundaries and initial uniform concentration $C(x, y, z, t=0)=$ $C_{0}$. In this case, with no scalar gradients, sources or sinks, the analytical solution if the flow field is divergence-free is $C(x, y, z, t)=C_{0}$. Figure 4 shows a $x z$ plane of the scalar concentration $C / C_{0}$ after $3 \times 10^{3}$ timesteps $(1,200 \mathrm{~s})$ using the trigonometric interpolation. It is clear that the non-conservativeness of the finite-volume discrete velocity field introduces spurious oscillations. As expected, the problem is worse closer to the bottom boundary. Although 

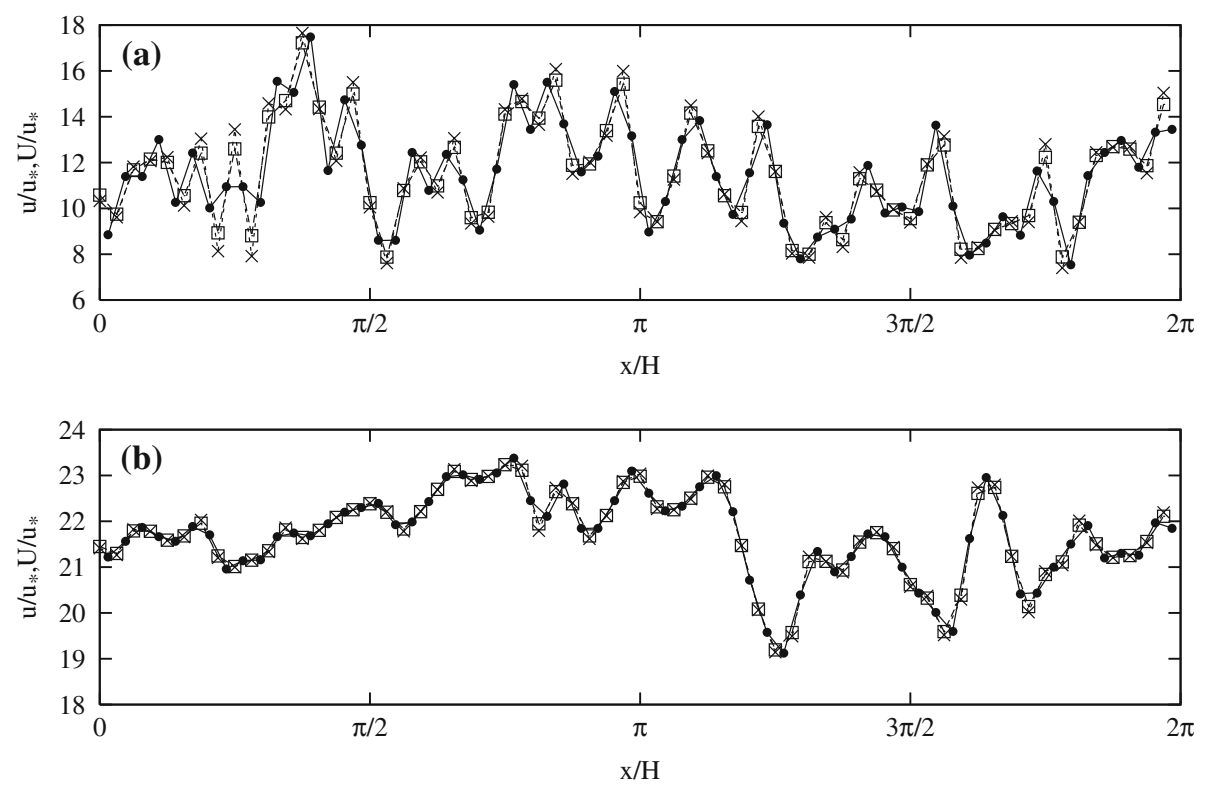

Fig. 3 Comparison between trigonometric and conservative interpolations for (a) first vertical grid point and (b) level $N_{z} / 4$. Original data (filled circles), trigonometric interpolation (open squares) and conservative interpolation (times)

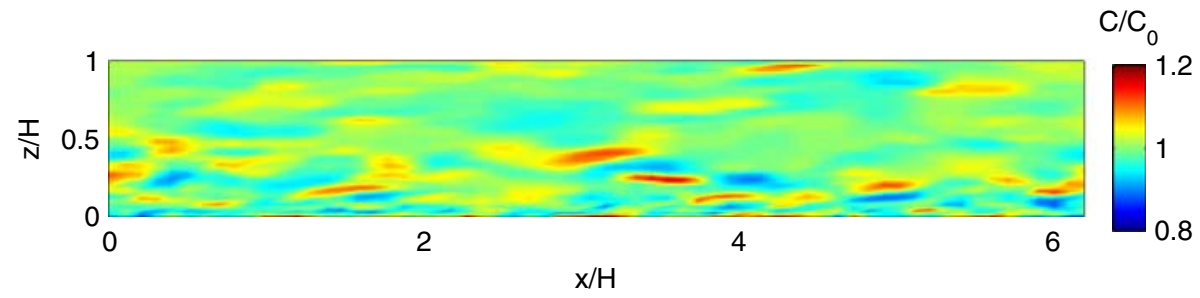

Fig. 4 Scalar concentration $x z$-cut of simulation using trigonometric interpolation, illustrating unphysical fluctuations developing due to artificial divergence

the volume integral of $C$ is conserved, the spurious oscillations have a large impact on the second-order moments, including variance and scalar fluxes. The time evolution of the scalar variances are shown in Fig. 5 for both interpolations at three different distances from the wall.

The second set of simulations has zero initial concentration $(C(x, y, z, t=0)=0)$ and a constant surface scalar flux imposed $\phi_{0}$ (again with zero scalar flux at the top). This case mimics the typical conditions assumed in the derivation of the Monin-Obukhov Similarity (MOS), which is a standard test for atmospheric boundary-layer simulations. Although there is no statistical steady state for the concentration field with these boundary conditions, the MOS theory predicts a logarithmic law for the normalized concentration difference (e.g. Monin 1970; Högström 1988)

$$
\frac{\left\langle C(z)-C_{r}\right\rangle}{C_{*}}=-\frac{S c}{\kappa} \log \left(\frac{z}{z_{r}}\right)
$$




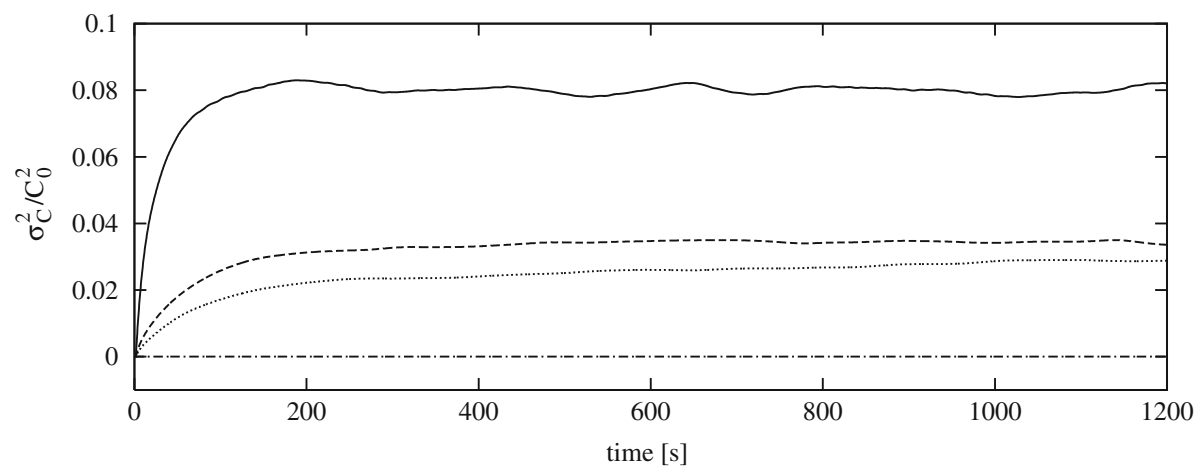

Fig. 5 Scalar variance evolution using trigonometric interpolation for plane $z=d z / 2$ (solid line), $z=H / 4$ (dashed line) and $z=H / 2$ (dotted line), and using conservative interpolation for any vertical plane (dot-dashed lines at $\left.\sigma_{C}^{2} / C_{0}^{2}=0\right)$
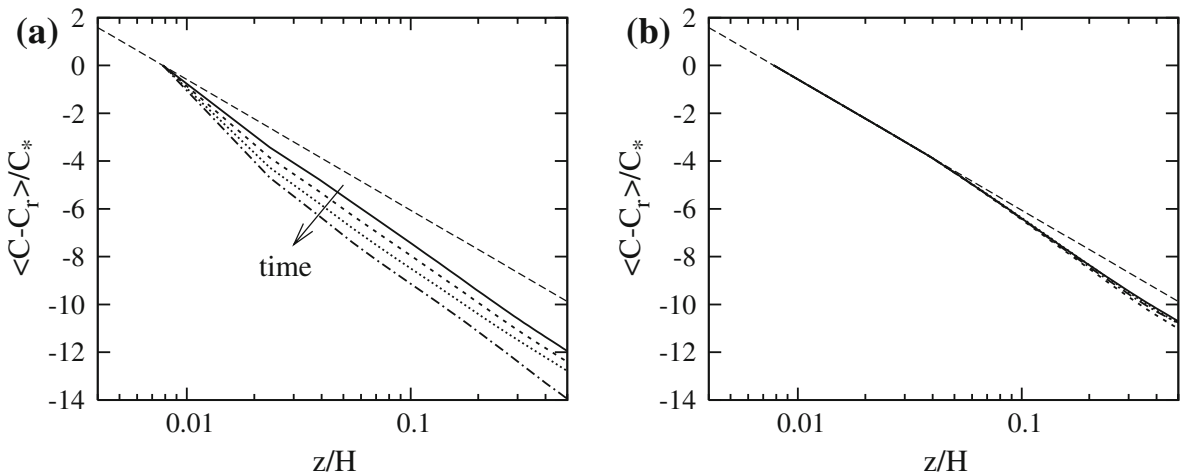

Fig. 6 Scalar vertical profile using (a) trigonometric interpolation and (b) conservative interpolation. Theoretical prediction (thin dashed line), LES time evolution averaged between $2.4 \times 10^{4}-4.8 \times 10^{4}$ (solid line), $7.2 \times 10^{4}-9.6 \times 10^{4}$ (dashed line), $1.2 \times 10^{5}-1.44 \times 10^{5}$ (dotted line) and $1.68 \times 10^{5}-1.92 \times 10^{5}$ (dot-dashed line)

where brackets represent an ensemble (or time) average, $C_{r}=C\left(z_{r}\right), z_{r}$ is a reference level taken here as the first vertical grid point, $C_{*}=\phi_{0} / u_{*}$ is a scale for the scalar concentration, $u_{*}$ is the friction velocity.

The evolution of Eq. 7 is shown in Fig. 6 for the simulations using the two interpolation schemes. The time averages are performed over $2.4 \times 10^{4}$ steps $(9,600 \mathrm{~s})$ and are shown for several intervals between $2.4 \times 10^{4}$ and $2 \times 10^{5}$ timesteps. It is very clear that not only the case with trigonometric interpolation does not agree with Eq. 7, but also a steady state is never reached despite the very long time integration performed. On the other hand, if the conservative interpolation is used the agreement is very good up to $z / H=0.1$ (note that MOS theory is only valid in the lowest $10 \%$ of the boundary layer).

The consequences of using non-conservative interpolations are even worse for the secondorder moments. The time evolution of the scalar variance profile is shown in Fig. 7. Clearly the variance increases monotonically in time reaching unphysically large values close to the ground surface if trigonometric interpolation is used. Results are improved if the conservative interpolation is used and results agree well with several other LES codes compared by 

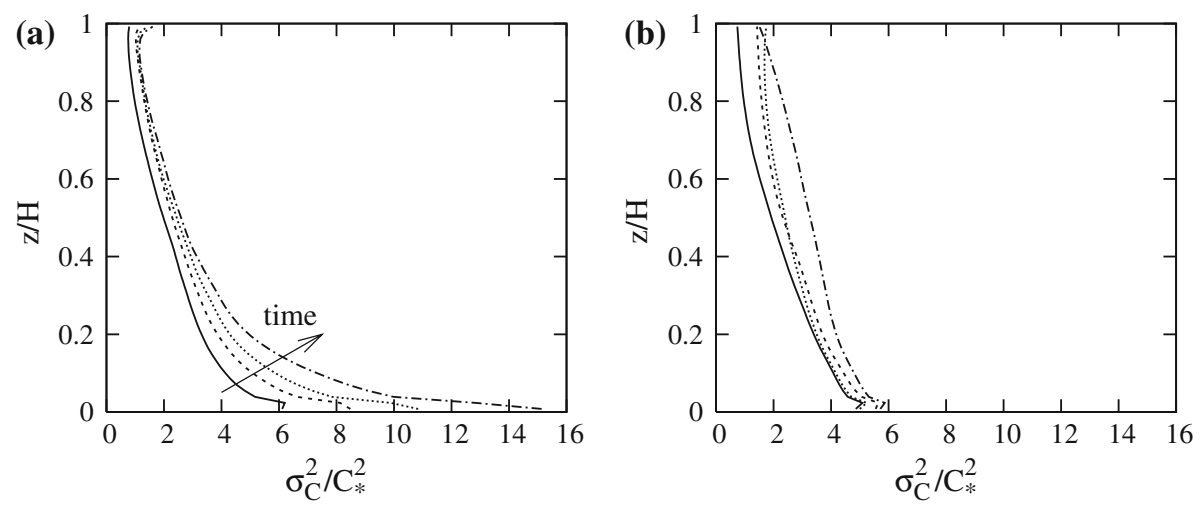

Fig. 7 Scalar variance profile (see Fig. 6 for legend)
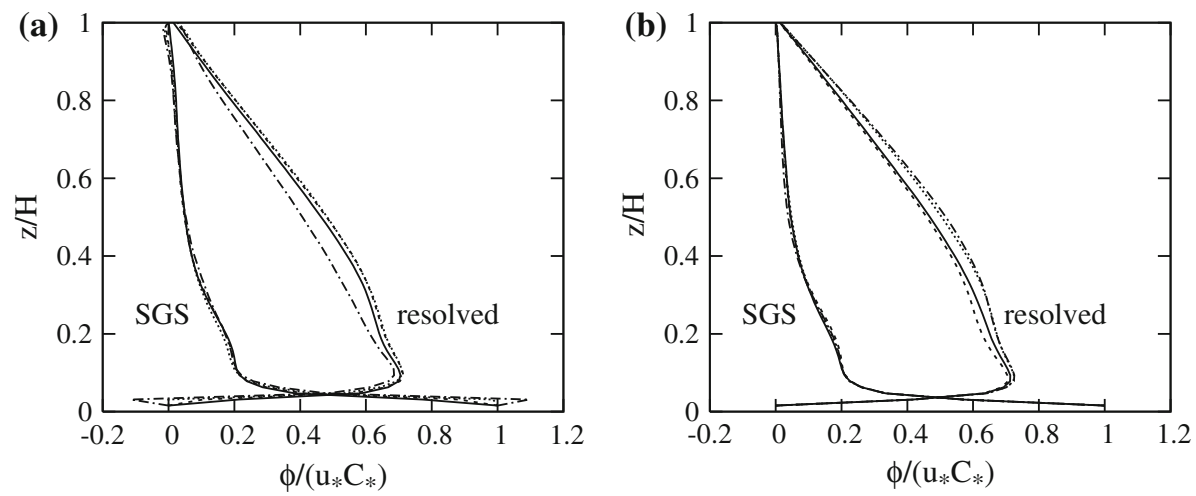

Fig. 8 Scalar vertical flux profile (see Fig. 6 for legend)

Andren et al. (1994). The vertical fluxes of the scalar field are presented in Fig. 8. Note that the trigonometric interpolation causes errors close to the surface to the point that the net resolved flux becomes negative (i.e. downwards) even though the vertical gradient is negative. Again no statistical steady state is reached. If the conservative interpolation is used, a steady state is reached, again in good agreement with results presented by Andren et al. (1994).

The vertical profiles of the SGS scalar diffusivity are shown in Fig. 9. It is clear that in both cases the modelled scalar diffusivity is very similar and cannot be the cause of the discrepancies shown above.

It is important to emphasize that in both simulations described above, a pseudospectral representation of the scalar field would yield good results. In the first case there are no sources and in the second the source is homogeneous in the horizontal directions. The main point of these examples is to illustrate the importance of enforcing the divergence-free conditions on the interpolated grid if a hybrid approach is to be used. Finally, to confirm the advantage of using a bounded discretization for the passive scalar as opposed to a pseudospectral representation in the case of isolated sources, the same simulation shown in Fig. 2 is now shown in Fig. 10 using the SMART discretization with the proposed interpolation. The general trends are similar but regions of low concentration that in Fig. 2 became negative and unphysical due to Gibbs oscillations, remain positive in Fig. 10 due to the SMART discretization. The 

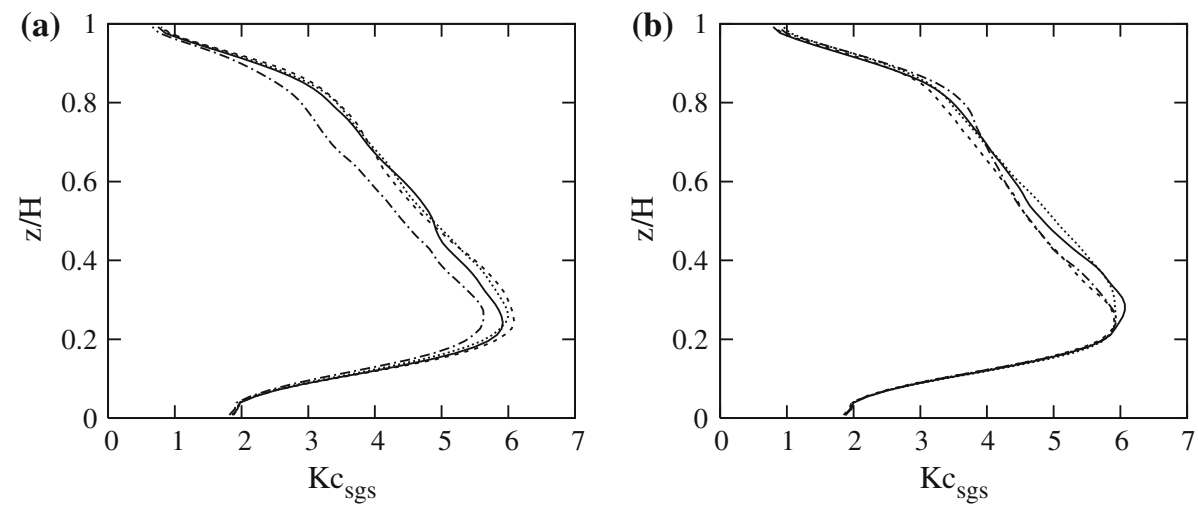

Fig. 9 Scalar SGS diffusivity profile, defined as $K c_{s g s}=v_{s g s} / S c$ (see Fig. 6 for legend)

(a)

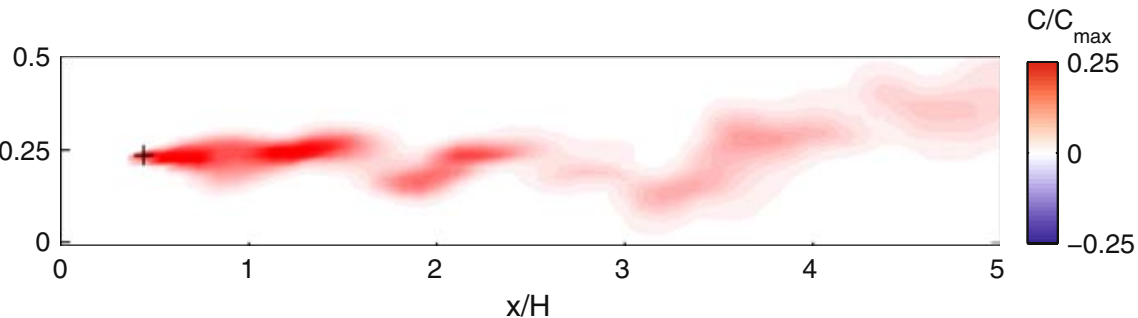

(b)

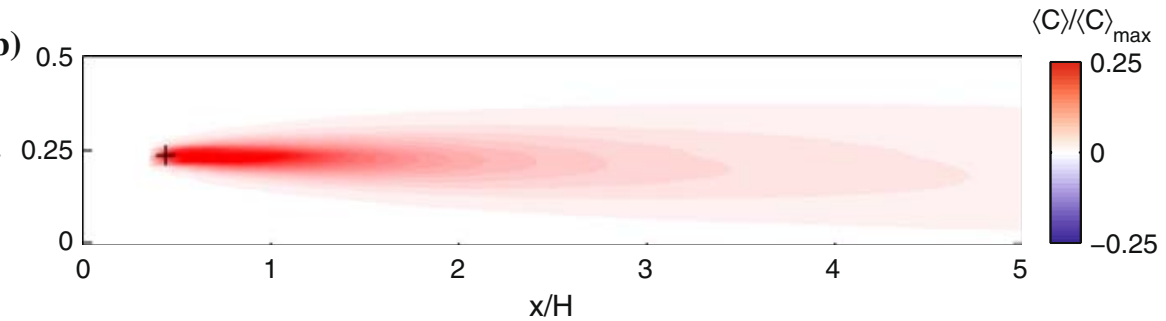

Fig. $10 x z$-cut of simulation of a point-source emission using SMART discretization and the conservative interpolation: (a) instantaneous concentration and (b) time-averaged concentration over $2.7 \times 10^{4}$ steps $(3 \mathrm{~h})$. The cross indicates the location of the source

time-average concentration plume also displays a much better behaviour with no oscillations upstream of the source.

\section{Conclusions}

In this note we illustrate the well-known drawbacks of using a pseudospectral representation for passive scalars when the sources are localized in space. In order to maintain the advantages of the high accuracy of the pseudospectral approach for the velocity field, a hybrid approach that combines a pseudospectral solution for the velocity field and a finite volume discretization for the passive scalar is proposed. Results presented here illustrate the importance of using a velocity field that obeys the divergence-free condition with high precision 
when interpolated onto the finite-volume grid. Typical interpolation schemes do not satisfy this condition, with negative consequences. A new conservative interpolation scheme has been proposed and the results are shown to be in agreement with theoretical predictions. The proposed method can be implemented efficiently and has the advantage of avoiding coupling between different velocity components (note that any attempt to use trigonometric interpolation and then correct the velocity field to satisfy continuity would yield a large system of equations coupling all velocity components). Although the illustrative example presented here is for a two-dimensional interpolation, extension to the three-dimensional case (e.g. where $(\partial w / \partial z)^{s p e c}$. would be known from a spectral representation) is straightforward.

Acknowledgements We acknowledge funding from the National Science Foundation biocomplexity project BES-0119903 and EAR-0609690.

\section{References}

Albertson JD, Parlange MB (1999) Surface length-scales and shear stress: implications for land-atmosphere interaction over complex terrain. Water Resour Res 35:2121-2132

Andren A, Brown AR, Graf J, Mason PJ, Moeng CH, Nieuwstadt FTM, Schumann U (1994) Large-eddy simulation of a neutrally stratified boundary layer: a comparison of four computer codes. Quart J Roy Meteorol Soc 120:1457-1484

Balsara DS (2001) Divergence-free adaptive mesh refinement for magneto-hydrodynamics. J Comp Phys 174:614-648

Basu S, Porté-Agel F, Foufoula-Georgiou E, Vinuesa JF, Pahlow M (2007) Revisiting the local scaling hypothesis in stably stratified atmospheric boundary-layer turbulence: an integration of field and laboratory measurements with large-eddy simulations. Boundary-Layer Meteorol 119:473-500

Bochev P, Shashnov M (2005) Constrained interpolation (remap) of divergence-free fields. Comput Methods Appl Mech Eng 194:511-530

Bou-Zeid E, Meneveau C, Parlange MB (2005) A scale-dependent lagrangian dynamic model for large eddy simulation of complex turbulent flows. Phys Fluids 17:025,105

Canuto C, Hussaini MY, Quarteroni A, Zang TA (1988) Spectral methods in fluid dynamics. Springer-Verlag, Berlin, $567 \mathrm{pp}$

Carey GF, Bicken G, Carey V, Berger C, Sanchez J (2001) Locally constrained projections on grids. Int J Numer Methods Eng 50:549-577

Gaskell PH, Lau AKC (1988) Curvature-compensated convective transport: SMART, a new boundednesspreserving transport algorithm. Int J Numer Methods Fluids 8:617-641

Högström U (1988) Non-dimensional wind and temperatures profiles in the atmospheric surface layer: a reevaluation. Boundary-Layer Meteorol 42:55-78

Khanna S, Brasseur JG (1997) Analysis of Monin-Obukhov similarity from large-eddy simulation. J Fluid Mech 345:251-286

Kosovic B, Curry JA (2000) A large eddy simulation study of a quasi-steady, stably stratified atmospheric boundary layer. J Atmos Sci 57:1052-1068

Kumar V, Kleissl J, Parlange MB, Meneveau C (2006) A large-eddy simulation of a diurnal cycle of the turbulent atmospheric boundary layer: atmospheric stability and scaling issues. Water Resour Res 42:W06D09

Li S, Li H (2004) A novel approach of divergence-free reconstruction for adaptive mesh refinement. J Comp Phys 199:1-15

Mao S, Feng ZG, Michaelides EE (2007) Large-eddy simulation of low-level jet-like flow in a canopy. Environ Fluid Mech 7:73-93

Moeng CH (1984) A large-eddy-simulation model for the study of planetary boundary-layer turbulence. J Atmos Sci 41:2052-2062

Monin AS (1970) The atmospheric boundary layer. Annu Rev Fluid Mech 2:225-250

Nieuwstadt FTM, de Valk JPJMM (1987) A large eddy simulation of buoyant and non-buoyant plume dispersion in the atmospheric boundary layer. Atmos Environ 21:2573-2587

Smagorinsky J (1963) General circulation experiments with the primitive equations. Mon Wea Rev 91:99-164

Tseng YH, Meneveau C, Parlange MB (2006) Modeling flow around bluff bodies and predicting urban dispersion using large eddy simulation. Environ Sci Tech 40:2653-2662 
Waterson NP, Deconinck H (2007) Design principles for bounded higher-order convection schemes—a unified approach. J Comp Phys 224:182-207

Xie Z, Hayden P, Voke PR, Robins AG (2004) Large-eddy simulation of dispersion: comparison between elevated and ground-level sources. J Turbulence 5:1-16

Yeung PK, Pope SB (1988) An algorithm for tracking fluid particles in numerical simulations of homogeneous turbulence. J Comp Phys 79:373-416

Yue W, Parlange MB, Meneveau C, Zhu W, van Hout R, Katz J (2007) Large-eddy simulation of plant canopy flows using plant-scale representation. Boundary-Layer Meteorol 124:183-203 